\title{
The role of lexical-semantic neighborhood in object naming: implications for models of lexical access
}

\author{
Tobias Bormann* \\ Department of Neurology, University Hospital Freiburg, Freiburg, Germany
}

\section{Edited by:}

Bradford Mahon, University of

Rochester, USA

Reviewed by:

Rasha Abdel Rahman, Humboldt

University, Germany

Gary Oppenheim, University of Illinois

at Urbana-Champaign, USA

*Correspondence:

Tobias Bormann, Department of Neurology, University Hospital

Freiburg, Breisacher Straße 64,

D-79106 Freiburg, Germany.

e-mail: tobias.bormann@uniklinik-

freiburg.de
The role of lexical-semantic neighborhood is relevant to models of lexical access. Recently it has been claimed that the size of the cohort of activated competitors affects ease of lexical selection in word production as well as the effect of semantically related distractors in picture-word interference tasks. Three experiments are reported in which subjects had to name pictures from large and small semantic categories (cf. "lion," "hammer" versus "funnel," "cage"). In Experiment 1, naming-impaired subjects exhibited semantic errors for targets from large categories. No semantic but many omission errors occurred for targets from small categories suggesting that few competitors were available for these "low competition targets." In contrast in two experiments with unimpaired subjects, targets were named equally fast. These experiments were sensitive enough to yield a highly significant repetition effect in Experiment 2. Contrary to the explicit predictions of a recent proposal, semantically related distractors caused interference for both groups of words in Experiment 3. The results suggest no role of neighborhood size in the naming of unimpaired individuals. Implications for models of lexical selection are discussed.

\section{INTRODUCTION}

Most models of lexical access in speech production assume that lexical selection is a competitive process (e.g., Levelt et al., 1999; Roelofs, 2001; Bloem and La Heij, 2003; Howard et al., 2006). That means, selection of a target word is influenced by the activation of competitors in the mental lexicon, usually other entries sharing semantic features with the target word. Activation of competitors makes selection of the intended lexical entry more difficult and may lead to a delay in naming. Other models of lexical access do not include competition. In these models, the lexical item with the highest level of activation is selected independently of the activation states of competing items (e.g., Dell, 1986). In both groups of models lexical errors occur when another lexical entry receives more activation than the target word.

One of the standard paradigms to study lexical access is the picture-word interference (PWI) experiment. This paradigm is a variant of the Stroop task (Stroop, 1935). Subjects name objects presented to them on a computer screen while a distractor word is also presented. The distractor slows down naming in comparison to a control condition (e.g., no distractor, or a line of "XXXX"). The semantic interference effect (SIE) refers to the observation that naming is slowed down more by distractors which come from the same semantic category to the target word (semantically related distractor) than a distractor from a different semantic category (unrelated distractor). For example, the naming of the target word "tiger" is affected by the semantic distractor "lion" more than by the unrelated distractor "letter." Most researchers have interpreted this as evidence for competition during lexical selection. The competitor in the lexical network receives activation, increases competition with the target and, thus, hinders its selection.

In a series of experiments, Mahon et al. (2007), have called into question this interpretation of the SIE and its relevance for the study of lexical selection. These authors provided a number of findings incompatible with the original "selection-by-competition" account (e.g., Miozzo and Caramazza, 2003; Finkbeiner and Caramazza, 2006; Mahon et al., 2007; Janssen et al., 2008; Mahon and Caramazza, 2009). For example, Miozzo and Caramazza (2003) provided evidence that low frequency distractors cause more interference than high frequency distractors. Finkbeiner and Caramazza (2006) observed semantic facilitation instead of interference when distractors were masked. Mahon et al. (2007), finally, observed stronger interference effects for related distractors which were dissimilar to the target word (e.g., "whale" as the distractor for "horse"). Under the "selection-by-competition" account, similar distractors should produce stronger competition because they share more features with the target. The authors' alternative account of interference effects in the PWI is the "response exclusion hypothesis." In short according to these authors, the PWI is of little value for studies of lexical selection. Instead, this paradigm involves a post-lexical decision mechanism controlling a limited-capacity response channel (cf. Ferreira and Pashler, 2002). In Stroop-like tasks, like the PWI, the distractor has privileged access to articulatory processes because reading is automatic and faster than picture naming. In the case of the PWI, the articulators must "first be disengaged from the articulators" (Mahon and Caramazza, 2009, p. 736) which delays the response. If distractors share relevant features with the target word, disengagement is slowed, thus, producing the interference effect.

Recently, some authors have set out to defend the "selectionby-competition" hypothesis. A detailed proposal is Abdel Rahman and Melinger's (2009a,b) "Swinging Lexical Network" hypothesis (henceforth: SLNH) which provides an explanation for interference and facilitation effects in PWI experiments, one of the central issues which need to be resolved (cf. Bloem et al., 2004; Finkbeiner and Caramazza, 2006). Abdel Rahman and Melinger draw attention to the number of co-activated competitors in determining 
ease of lexical access (cf. Roelofs, 1992, 2001). They argue that the number of activated competitors or, lexical neighborhood size, is a critical factor in lexical access and will determine whether a semantically related distractor will interfere with or will facilitate lexical selection.

The SLNH assumes that during object naming, two independent processes are triggered and need to be considered when evaluating facilitation and interference effects. First, there is activation spreading within a semantic network of related concepts which, in general, facilitates processing of the target concept. Second within the lexical network, that is, within the cohort of semantically related concepts, which compete for selection, the mutual activation causes activation spreading which hinders selection of the target item. Within the lexical network, activation spreading, thus, contributes to interference effects (Abdel Rahman and Melinger, 2009a, p. 715f.). When determining the net effects of facilitation and interference, the activation states of both the conceptual and the lexical network need to be considered. In the case of Mahon et al.'s (2007) semantic distance effects, the SLNH suggests that a semantically far distractor activated a larger set of competitors while a semantically close distractor activated a smaller set of coactivated competitors. In the case of the larger cohort, a distractor would cause more interference than in the case of a small cohort. Abdel Rahman and Melinger's account, thus, predicts that in case of competition through a distractor, there should be an interaction between neighborhood size and semantic relatedness of the distractor. This is because with broader semantic categories, the distractor should produce greater interference. "We assume that semantic contexts always induce both conceptual facilitation and lexical competition. The net effects will be facilitation-dominant when only a single or small number of competitors is activated, in which case conceptual priming outweighs lexical competition. In contrast, effects will be interference-dominant when a large number of inter-related competitors, a lexical cohort, is active, in which case cohort-induced lexical competition outweighs conceptual priming" (Abdel Rahman and Melinger, 2009a, p. 716-717). The same mechanisms can cause facilitation effects through distractors which are associatively related to the target or which involve a part-whole relationship: as these distractors contribute to facilitation through semantic activation spreading yet are part of a different lexical network, conceptual facilitation is much larger than interference through related lexical competitors. The assumption that interference in the lexical network depends on the number of activated lexical entries is shared with other models (e.g., Roelofs, 1992; La Heij and van den Hof, 1995; Levelt et al., 1999).

The SLNH was originally developed to explain interference and facilitation effects in PWI experiments but the two independent factors - conceptual facilitation and lexical interference - are the result of automatically triggered processes which should also be involved in other naming tasks. Hence, the authors suggest their model to be relevant for other "context" situations, for example, blocked naming experiments and the generalization of interference effects to other category members (Belke et al., 2005). In this study, repeated activation of a larger cohort of related lexical entries led to generalization to other, previously inactive lexical entries. Two predictions can be derived from this account: first, ceteris paribus, a larger cohort of co-activated lexical entries will activate each other and will, thus, produce more competition in the lexical network (e.g., Abdel Rahman and Melinger, 2009b, p. 751). This is because a large set of competitors will cause the lexical network to "resonate," namely to increase activation across the cohort which interferes with the selection process. The facilitation effect in these two different contexts should be comparable because this stems from automatic spread of activation along associatively related concepts. Second, the size of the activated cohort of competitors further determines whether a semantically related distractor word should interfere with naming or should facilitate lexical access. With a larger set of competitors, a semantically related distractor in a PWI experiment will again increase the overall activation of the network while, in contrast, in the case of few competitors or in the case of distractors from different categories, there will be less mutual activation and interference. The SLNH, thus, makes testable claims about the size of the semantic neighborhood in lexical selection. This account predicts that the number of co-activated semantically related competitors has a direct influence upon ease of lexical selection and should modulate the effects of semantically related distractors.

Previous research on neighborhood effects has focused on phonological and orthographic neighborhood. Studies suggest that formally related neighbors play different roles in word perception and word production. Phonologically related neighbors appear to interfere in spoken word recognition (and orthographically related neighbors interfere in reading) while they facilitate word production (see Dell and Gordon, 2003, for review). For example, Stemberger (2004) as well as Vitevitch (2002) reported fewer speech errors and faster naming latencies for words with many neighbors.

Fewer studies are available regarding semantic neighborhood size (though see Caramazza and Costa, 2001; Vigliocco et al., 2002). Lexical-semantic neighborhood size has recently been identified as a critical variable in the naming performance of anomic subjects by Blanken and colleagues (Blanken et al., 2002; Bormann et al., 2008). In a single case study, Blanken et al. (2002) demonstrated that for items from broad semantic categories with many competitors (e.g., "lion," "hammer"; "high competition target items") their anomic subject MW exhibited semantic errors. In contrast for items from small semantic categories ("cage," "funnel"; "low competition items"), semantic errors hardly ever occurred. Instead, MW exhibited errors of omission (i.e., no response at all). The study has been replicated by Bormann et al. (2008) for a group of 17 aphasics. The number of correct responses for both groups of items did not differ suggesting that overall, ease of access to the target lexical entry was comparable. A further replication was reported by Kittredge et al. $(2007)^{1}$. The data from the aphasic subjects strongly suggest that in the case of high competition items, a larger set of competitors is activated. In contrast, for low competition target items, the cohort is considerably smaller, or, a cohort of co-activated lexical entries may not even be available. In the case of high competition items, semantically related competitors may receive above threshold activation which leads to a semantic error.

There is now evidence in the literature suggesting comparable mechanisms underlying interference in aphasic naming errors and slowed naming latencies in unimpaired speakers. For example,

${ }^{1}$ Note that the replication was reported in the actual poster presentation and not in the published abstract which included fewer subjects and analyses. 
Schnur et al. (2006) demonstrated that unimpaired speakers' response times increased in blocked naming in comparison to the mixed condition. Aphasic subjects exhibited an increase of semantic errors in blocked naming tasks. Several other studies have demonstrated that aphasics produce more semantic errors in homogenous contexts (e.g., Laine and Martin, 1996) while unimpaired speakers exhibit an increase in naming latencies (Howard et al., 2006; Navarrete et al., 2010).

The present study set out to assess the central claims of the SLNH, namely, that (1) semantic neighborhood size influences speed of lexical selection and that (2) it interacts with distractor interference. The theory makes two claims: it predicts a main effect of neighborhood density since naming latencies for items from larger semantic categories should be slower due to increased competition for selection. This prediction was tested in an experiment in which subjects were asked to name items in three consecutive cycles. Repetition of a target item speeds up naming in the subsequent presentation cycle (e.g., Ellis et al., 1996; Barry et al., 2001; Belke et al., 2005). The second prediction of Abdel Rahman and Melinger's (2009a) hypothesis is that of an interaction of distractor type and lexicalsemantic neighborhood size: for high competition targets, a semantically related distractor should slow down naming (in comparison to an unrelated distractor). In contrast, for low competition target items, a related distractor should facilitate and, thus, speed up naming (see above). This prediction was tested in a PWI experiment.

\section{MATERIALS AND METHODS SELECTION OF MATERIALS}

A subset of Bormann et al.'s (2008) target items was used in the present study, 54 target items which had few or many competitors (27 per group). The difference in CELEX frequency (Baayen et al., 1993), age of acquisition, length (no. of syllables, no. of letters), and picture name agreement was assessed using $t$-tests for independent samples. To control for different phonetic onsets, response time residuals (in milliseconds) for each word's initial phoneme were taken from Kessler et al. (2002; Table 4, p. 155). These authors provide residual response times for each onset phoneme after excluding covariables (including frequency and length). No significant differences were observed in all these variables (Table 1).

In the original error corpus of Bormann et al. (2008), there was a total of 68 semantic errors for the high competition targets, that were targets in the present study, and 13 semantic errors for the low competition targets to be used in the present studies. In the high competition item group, only $6 \%$ of semantic errors had been associatively related $(n=4)$ while in the low competition group, $23 \%$ of semantic errors were associative errors (3 out of 13). All other errors were co-ordinated semantic errors. Thus, only a small fraction of aphasic misnamings were associatively related to the target. The target words for both experiments are presented in the Appendix.

Pictures were gray-scale bitmaps and were formatted to a size of $9 \times 9 \mathrm{~cm}$. To control for item recognition times, 10 subjects not participating in the naming experiments were asked to verify the pictures. They were first presented the pictures along with 54 foils. In the recognition experiment, a fixation cross appeared on the computer screen followed by a word. This was followed by a picture which could either match the word or not. The 54 target
Table 1 | Features of target words with many and few competitors.

\begin{tabular}{|c|c|c|c|c|}
\hline & $\begin{array}{l}\text { Many } \\
\text { competitors }\end{array}$ & $\begin{array}{l}\text { Few } \\
\text { competitors }\end{array}$ & $\begin{array}{l}t \text {-Value } \\
(d f=52)\end{array}$ & $p$-Value \\
\hline Word frequency & 74.33 & 80.30 & -0.20 & $p>0.84$ \\
\hline Age of acquisition ${ }^{a}$ & 2.61 & 2.95 & -1.62 & $p>0.11$ \\
\hline Length - syllables & 1.81 & 1.81 & 0.00 & $p>0.99$ \\
\hline Length - letters & 5.59 & 5.78 & -0.57 & $p>0.56$ \\
\hline Name agreement ${ }^{a}$ & 19.18 & 19.81 & -1.65 & $p>0.10$ \\
\hline $\begin{array}{l}\text { Picture recognition } \\
\text { time (in ms) }\end{array}$ & 522.1 & 518.1 & 0.26 & $p>0.79$ \\
\hline $\begin{array}{l}\text { Phonetic response } \\
\text { time residuals }\end{array}$ & 4.20 & 6.65 & -0.42 & $p>0.67$ \\
\hline $\begin{array}{l}\text { Mean rated } \\
\text { competitiveness }\end{array}$ & 4.67 & 2.54 & $9.29^{c}$ & $p<0.001$ \\
\hline
\end{tabular}

aFrom Bormann et al. (2008).

${ }^{b}$ Kessler et al. (2002; Table 5).

${ }^{c}$ Corrected degrees of freedom $=47.04$, due to unequal variances.

items were preceded by the correct word ("yes"-responses) whereas 54 filler items followed a different word ("no"-responses). Subjects were instructed to respond as quickly as possible by button press. Presentation of items and response latency measure was controlled by DMDX (Forster and Forster, 2003). Only correct positive responses were assessed. The recognition experiment revealed that items from both groups could be verified equally quickly.

Finally, to assess the target items' competitiveness independently of the original study, a further group of naïve raters judged the semantic competitors of the target items. These raters, who were not involved in any of the other studies, judged the competitiveness of the items on a seven-point scale. Twelve advanced students of linguistics of the University of Freiburg (third year) were presented the target items and were asked to judge whether an item had "very many" (corresponding to the "seven" on the seven-point scale) or "no or almost no" co-ordinated competitor(s) (corresponding to the "one" on the scale). They were given an instruction on lexical-semantic competition as well as several examples. The respective mean judgments were calculated and assessed with a $t$-test for independent samples. There was a highly significant difference between low and high competition targets with respect to their rated competitiveness (also listed in Table 1). The study of Bormann et al. (2008) had failed to provide quantitative measures of competitiveness. This short-coming was fixed here. The naïve raters gave significantly different ratings of competitiveness of the involved items ${ }^{2}$.

Kittredge et al. (2007), in their replication of the study of Bormann et al. (2008), adopted a different approach based on latent semantic analysis (LSA; Landauer et al., 1998). They counted the concepts which were members of the same semantic category and

${ }^{2}$ The judgment of competitiveness through naïve raters was carried out after Experiments 2 and 3 had been conducted but prior to Experiment 1. The results were evaluated independently, albeit informally, by asking four additional naïve subjects, two being experienced linguists, to produce competitors in response to the target words of the two conditions. Subjects produced an average of 8.29 (SD 3.01) competitors for the high and 1.41 (SD 1.45) competitors for the low competition targets. 
had a similarity to the target of 0.40 or higher. They also analyzed the original target items of Bormann et al. (2008) and reported significant differences for these items.

In addition, the distractors to be used in Experiment 3 were rated for competitiveness since the distractors in a PWI experiment activate parts of the conceptual and lexical network. The distractors for the high competition target words received significantly higher mean ratings for competitiveness compared to the low competition distractors $(t=-5.47, p<0.01)$. Thus in Experiment 3, there were two conditions, high competition target items with high competition distractors versus low competition targets with low competition distractors.

\section{EXPERIMENT 1}

\section{Participants}

A group of subjects $(n=7)$ with impaired naming participated. All subjects presented with progressive anomia with errors of omission and lexical errors. None of the subjects exhibited phonemic errors in naming and repetition. Five subjects received the diagnosis of semantic dementia, one presented with symptoms of both word finding difficulties and behavioral changes (suspected behavioral variant of FTLD), one subject received the diagnosis of probable Alzheimer's disease. The diagnosis was made by a neurologist based on neuropsychological profile, structural MR imaging, and PET scans. The semantic dementia patients presented with profound anomia in the face of good episodic memory. This group is particularly suited to address the present questions as these subjects usually do not exhibit phonemic errors. It has been demonstrated that the naming deficit in both conditions, semantic dementia as well as Alzheimer's type dementia, consists of a deficit of lexical access from semantics (e.g., Hodges et al., 1996; Lambon Ralph et al., 1998). In their naming, the subjects usually exhibit effects of familiarity, frequency, and age of acquisition but no effect of word length (e.g., Lambon Ralph et al., 1998).

\section{Materials}

There were 27 target items in each group which were selected to be suited for reaction time studies (Experiments 2 and 3 ).

\section{Procedure}

The subjects participated in an untimed naming experiment in which the pictures were presented on a computer screen. Beginning of picture presentation was marked by a short tone, sessions were digitally recorded. Sessions were transcribed with the first response within $10 \mathrm{~s}$ being classified as a correct response, a (co-ordinated) semantic error, an omission error (including circumlocutions; Dell et al., 2004) or "other" responses (picture misidentification; phonemic error; proper name; associatively related semantic error).

\section{Results}

The number of correct responses, semantic errors, as well as omissions (Table 2) for both groups of target items was compared by means of two $t$-tests (with subjects and items as random factors). The number of correct responses did not differ significantly suggesting comparable levels of difficulty for the two groups of items (by subjects: $t<1.0$; by items: $t<1.0$ ). The number
Table 2 | Mean numbers of correct responses and errors in Experiment 1.

\begin{tabular}{lcc}
\hline & $\begin{array}{l}\text { High competition } \\
\text { targets }\end{array}$ & $\begin{array}{l}\text { Low competition } \\
\text { targets }\end{array}$ \\
\hline Mean no. correct & 13.57 & 13.43 \\
Mean no. semantic errors & 7.43 & 0.71 \\
Mean no. omissions & 5.71 & 12.14 \\
Mean no. other errors & 0.29 & 0.71 \\
\hline
\end{tabular}

of semantic errors was higher for the high competition targets (by subjects: $t=5.85, p<0.01$; by items: $t=-4.55, p<0.01$ ). In contrast, the number of omission errors was higher for the low competition targets (by subjects: $t=-4.5, p<0.01$; by items: $t=3.69, p<0.01)$. Only three of the participants' responses were phonemic errors ( $<1 \%$ of all responses). This suggests that the main impairment was in accessing the intended word form rather than a deficit in post-lexical phonological encoding (cf. Butterworth, 1992). There was a greater variety of semantic errors in the high competition group. Only 5 responses in the low competition group were semantic errors $(2.5 \%)$ while 52 responses in the high competition group were semantic errors $(27.5 \%)$. The five semantic errors in the low competition group occurred for five different target words. In contrast, in the high competition group, there were between one and four different semantic errors (e.g., target "fox" - monkey, dog, wolf, bear) with a mean of 1.7 different responses.

\section{Discussion}

Experiment 1 provides a full replication of Bormann et al.'s (2008) and Kittredge et al.'s (2007) results: for anomic subjects, lexicalsemantic neighborhood determined the type of error response. Target items from large semantic neighborhoods provoked semantic errors. In contrast, the target items from small neighborhoods provoked a smaller number of semantic errors but a larger number of omission errors. While the difference for the semantic errors was expected, the different number of omission errors is particularly interesting. It suggests that omission errors, thought to occur at the level of word form access (Goodglass et al., 1976; Levelt et al., 1999), are affected by semantic neighborhood and, thus, activation states at the lemma level in Levelt et al.'s (1999) model.

Central to the present study, the results suggest that items from large semantic neighborhoods were exposed to a larger set of competitors compared to the target words from small neighborhoods. It is relevant at this point that only a small fraction of word errors in the original study as well as the present assessment consisted of associatively related words. Associatively related errors were included in the "other errors"-category, and there was less than one "other" error per subject. This provides evidence that competitors during naming were category co-ordinates. In contrast, associated concepts do not appear to activate their respective lexical entries to engage in competition. The replication of the original study was successful with a subset of the original target items and a different subject group. The participants suffered from progressive naming impairment in the context of semantic dementia or 
probable Alzheimer's disease, not from stroke aphasia. The main goal, however, was to demonstrate that the set of target items to be employed in the next naming experiments provoke different errors: the highly competitive items provoke semantic errors while the low competition items do not. A reasonable explanation for this is that high competition items are exposed to a larger number of competitors than targets from small categories. According to the "Swinging Lexical Network" hypothesis, this should affect speed of lexical access in unimpaired speakers which was evaluated in the next two experiments.

\section{EXPERIMENT 2}

\section{Participants}

Eighteen undergraduate students as well as staff from the department (mean age 29.8 years) took part in the experiment and were played five euros for participation. All were native speakers of German and had normal or corrected-to-normal vision. None had any history of mental illness. All were naïve regarding the purpose of the study.

\section{Procedure}

Prior the naming experiment, subjects were presented all 54 pictures on the screen and were asked to name the pictures aloud. The name to be used in the experiment was then presented above the picture. In the naming experiment, subjects were asked to name the pictures as quickly as possible. Subjects were seated about $60 \mathrm{~cm}$ from a 17 computer screen and wore a headset with a microphone connected to a computer. Presentation of items was well as response latency measure was controlled by DMDX (Forster and Forster, 2003). Items were presented in three cycles, each item was presented three times, once in each cycle. Order of presentation was randomized within each cycle with the constraint that there were at least 20 pictures between items from the same semantic category (e.g., "seagull" and "swan"). Subjects were instructed to produce the names of the objects as quickly as possible, to speak in a constant voice and not to make any noise apart from the object name. The microphone was positioned in a fixed distance to the mouth (cf. Kessler et al., 2002).

\section{Results}

Four types of responses were excluded from the analysis: (1) responses different from the intended target word, (2) verbal disfluencies and self-corrections, (3) microphone failures. This led to $5.2 \%$ responses being excluded. In addition, (4) responses with latencies above an individual cut off were excluded. For each subject, the cut off was determined by calculating the subject's mean response latency across all conditions and adding 2.5 SD. A further $2.7 \%$ of responses were excluded. We assessed the factors "neighborhood size" and "presentation cycle." Naming latencies were averaged and were submitted to analyses of variance with subjects and items as random factors ( $F_{1}$-analysis; $F_{2}$-analysis). Mean response latencies as well as SD are provided in Table 3.

The main effect of presentation cycle was highly significant with faster responses in cycle 2 and $3\left[F_{1}(2,34)=37.31, p<0.01\right.$; $\left.F_{2}(2,104)=82.99, p<0.01\right]$. The second factor, semantic neighborhood size, was not significant $\left[F(1,17)=1.597, p>0.2 ; F_{2}(1\right.$, $52)=0.03]$. There was no interaction between these two factors
Table 3 | Mean naming latencies and errors across cycles and conditions (in ms; SD in parentheses).

\begin{tabular}{lllll}
\hline & & Cycle 1 & Cycle 2 & Cycle 3 \\
\hline Many neighbors & RT & $685(66)$ & $636(59)$ & $624(54)$ \\
& Errors & $0.55(0.78)$ & $0.39(0.61)$ & $0.17(0.38)$ \\
Few neighbors & RT & $697(68)$ & $636(57)$ & $628(69)$ \\
& Errors & $0.56(0.70)$ & $0.56(0.51)$ & $0.0(0.0)$ \\
\hline
\end{tabular}

$\left[F_{1}(2,34)=1.23, p>0.29 ; F_{2}(2,104)=0.31, p>0.7\right]$. The main effect of presentation cycle was significant because of the difference between the first and the second presentation, but the comparison of the second and the third block failed to reach significance. The error analysis revealed a significant effect of cycle which was significant in the $F_{1}$-analysis $\left[F_{1}(2,34)=10.96, p<0.01 ; F_{2}(2,104)=2.18\right.$, $p<0.12$ ] while the difference between conditions and the interaction were not significant $(F$ s $<1.0)$. With more repetitions, fewer errors occurred.

If target items were split along the median age of acquisition and response times were considered, an analysis of variance revealed a highly significant effect of presentation cycle $\left[F_{1}(2,34)=39.5\right.$, $\left.p<0.01 ; F_{2}(2,104)=84.95, p<0.01\right]$ and a highly significant effect of age of acquisition $\left[F_{1}(1,17)=109.73, p<0.01 ; F_{2}(1,52)=15.64\right.$, $p<0.01]$. The interaction between both factors was not significant. Thus, early acquired items were named faster than late acquired items. A linear mixed effects analysis (e.g., Baayen et al., 2008) revealed significant effects of "age of acquisition" $(t=2.53)$ as well as significant contrasts for cycle 1 versus $2(t=-3.94)$ and cycle 1 versus $3(t=-4.95)$. "Competition" was not significant in that analysis $(t=0.08)$, and the interaction of "cycle" and "competition" was far from significant $(t<0.5)$.

\section{Discussion}

Experiment 2 failed to find a main effect of lexical-semantic neighborhood size which is predicted by the "swinging network" account. There was no difference between the two groups of items. In fact, response times for the two item types were virtually identical. Across the three presentation cycles, the difference between the two groups of items was $5 \mathrm{~ms}$. In contrast, a repetition effect was found between the first and the second cycle which has been reported frequently (e.g., Barry et al., 2001; Belke et al., 2005). There was no interaction between the two factors. There was a trend for items in the third cycle to be named faster than in the second but this difference was not significant. We note that in the study of Belke et al. (2005), the effect of presentation cycle was largely driven by a drop of naming latencies in the second presentation cycle with little speed up thereafter. Therefore, it appears as if subjects quickly learn the mapping between a picture and the respective naming response and reach the maximum speed in the second cycle already. This, however, is not central to our argument which focuses on the difference between highly and low competition target words.

The experiment does not provide support for the central claim of the SLNH of Abdel Rahman and Melinger (2009a). According to this hypothesis, the size of a target word's lexical-semantic neighborhood should influence response latencies because for a 
larger set of competitors, selection should be more difficult. This, however, was not supported by the data. This is of relevance for other models of lexical access which either explicitly (Roelofs, 1992; Levelt et al., 1999) or implicitly (Caramazza, 1997; Bloem and La Heij, 2003) consider the number of activated lexical entries to determine ease of lexical selection. Previous studies had assessed the role of set size in the context of PWI experiments (e.g., La Heij and van den Hof, 1995) but not in single object naming experiments.

It should be noted that the significant effects of presentation cycle and age of acquisition suggest that the experiment was powerful enough to yield significant results in general. On the other hand, these effects are not easily comparable as repeated presentation of items as well as age of acquisition are probably more powerful effects than neighborhood size. On the other hand, the main effect of neighborhood size was far from significant. It is rather unlikely that the failure to find a significant effect resulted from a lack of statistical power due to a limited number of subjects as, first, there was not even a trend for "low competition" items to be named faster and, second, the $F$-value was even smaller in the analysis by items (with more degrees of freedom). In contrast, during the first presentation cycle, a numerical advantage for high competition items was observed. In the next experiment, we shall assess the role of neighborhood size in a classical PWI paradigm which will further allow for the assessment of interactions between neighborhood size and distractor type and, again, for a main effect of neighborhood size. This will be particularly relevant as the SLNH has as a focus the semantic context created by distractor words.

\section{EXPERIMENT 3}

\section{Participants}

A group of 24 undergraduate and graduate students as well as staff of the department took part in the study (mean age 27.8 years). All were native speakers of German with no known history of neurological or psychiatric disease. All had normal or corrected-tonormal vision, all were naïve regarding the purpose of the study. Five subjects were familiar with the classic Stroop paradigm and noted the similarity between the PWI task and the color Stroop task but all reported to have missed the difference between semantically related and unrelated distractors. None had participated in Experiment 2 and the recognition experiment.

\section{Materials}

The same items were used which had been used in Experiments 1 and 2. Distractor words were printed in red capitals in 22 point, bold Arial font, and were printed centrally across the picture. Distractor words were localized a maximum of $2 \mathrm{~cm}$ around the central fixation cross. All subjects reported that the distractor was impossible to ignore but that the picture could be identified easily.

For each target word, a semantically related distractor was found. Distractors in the two conditions were concrete nouns matched for length (5.1 versus 5.6 letters) and CELEX frequency (122.6 versus 131.3). $t$-Tests for independent samples revealed no differences for frequency and length of the distractors [frequency: $t(52)=-0.76$, $p>0.9$; length: $t(52)=-1.65, p>0.1]$. For the unrelated condition, the same distractors were paired with an unrelated target word. The distractor word was presented simultaneously with the target picture (stimulus onset asynchrony of $0 \mathrm{~ms}$ ).

\section{Procedure}

Subjects were first familiarized with the pictures. They were shown each of the 54 items along with six training items, and the intended name of the item. Before the experiment, six training trials were carried out in which the subjects had to name the pictures with the distractors or xxx printed across them. The training items had been presented in the familiarization procedure but did not appear as experimental items.

In the experiment, target items were presented with three types of distractors: a line of "Xxxx" (control condition), a semantically unrelated distractor or a semantically related distractor (see Appendix). Three blocks were presented with short breaks between them. In each block, each item was presented once, with one of the three distractors. Within each block, presentation was randomized with the same constraint as in Experiment 2. The number of xxx, related and unrelated distractors was balanced in each block. Between each block, subjects could rest and start the next block by hitting the spacebar. The session lasted about $20 \mathrm{~min}$. Presentation of the items was controlled by DMDX. The subjects wore a headset with a microphone connected to the input port of a computer, and response time was measured to the nearest millisecond.

\section{Results}

For the analysis of the naming latencies, four types of responses were excluded from the analysis: (1) responses different from the intended word, (2) self-corrections and disfluencies, (3) microphone failures $(5.8 \%)$. In addition, responses above an individual cut off (individual's mean $+2.5 \mathrm{SD}$ ) were excluded $(2.4 \%)$. Naming latencies were averaged across items $\left(F_{1}\right.$-analysis $)$ and across subjects $\left(F_{2}\right.$-analysis $)$ and were submitted to analyses of variance. The mean response latencies and error scores, along with their SD, are presented in Table 4 and Figure 1.

Mauchly's test revealed a violation of the sphericity assumption for the factor "distractor type" $\left[\chi_{(1)}^{2}=9.37, p<0.05\right]$, therefore the degrees of freedom were corrected using Greenhouse-Geisser estimates of sphericity $(\varepsilon=0.743)$. A distractor word slowed down naming latencies, and a semantically related distractor produced more interference than a semantically unrelated distractor. The main effect of "distractor type" was highly significant $\left[F_{1}(1.49\right.$, $\left.34.16)=80.08, p<0.01 ; F_{2}(2,104)=69.8, p<0.01\right]$. The second factor of "neighborhood size" failed to reach significance $\left[F_{1}(1\right.$, $\left.23)<1.0 ; F_{2}(1,52)<0.1\right]$. The interaction between both factors was significant in the by-subject analysis but failed to reach significance in the by-item analysis $\left[F_{1}(1.8,43.7)=3.43, p<0.05 ; F_{2}(2\right.$, $104)<1.0]$. As becomes clear from visual inspection of the data (Figure 1), the overall interaction effect resulted from an involvement of the neutral ("xxxx") condition. When only the response times for the related and unrelated distractor conditions were compared in a $2 \times 2$ ANOVA, the interaction was not significant $\left[F_{1}(1,23)<1.0 ; F_{2}(1,52)<1.0\right]$. The effect of "distractor" was highly significant $\left[F_{1}(1,23)=19.77, p<0.01 ; F_{2}(1,52)=11.06\right.$, $p<0.01]$ while the difference between target words with few and many competitors, again, was not significant $\left[F_{1}(1,23)=2.52\right.$, 
Table 4 | Mean naming latencies and errors across conditions (in ms, SD in parentheses).

\begin{tabular}{lllll}
\hline & & xxxxx & Unrelated & Related \\
\hline High competition & RT & $727(82)$ & $813(96)$ & $842(103)$ \\
& Errors & $0.54(0.88)$ & $0.46(0.59)$ & $1.25(1.19)$ \\
Low competition & RT & $743(94)$ & $808(103)$ & $829(101)$ \\
& Errors & $0.50(0.78)$ & $0.46(0.66)$ & $0.92(0.88)$
\end{tabular}

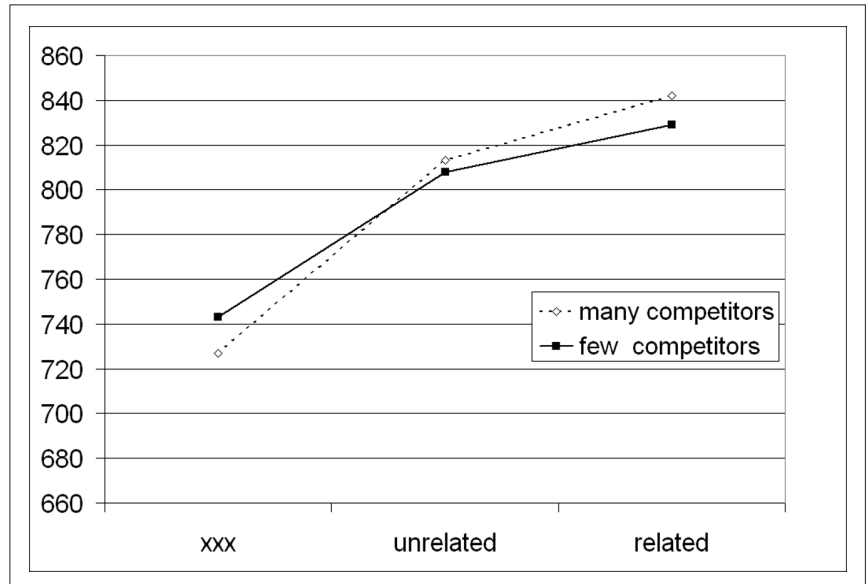

FIGURE 1 | Mean naming latencies across conditions (in ms).

$\left.p>0.12 ; F_{2}(1,52)=0.07\right]^{3}$. In order to get the interaction significant $(f=0.1534,1-\beta=0.80, r=0.5)$, a group of 86 subjects would have been required while in the by-item analysis, 5898 items would have been required to yield a significant interaction $(f=0.0316$, $1-\beta=0.80, r=0.5)$. Compare this to a required sample size of 5 subjects and 12 items for significant main effect of "distractor type" $(f=0.9229, f=0.4605 ; 1-\beta=0.80, r=0.5)$. The error analysis $(3 \times 2$ ANOVA) revealed a highly significant effect of distractor type $\left[F_{1}(2,46)=9.47, p<0.01 ; F_{2}(2,104)=6.66, p<0.01\right]$ with more errors occurring for related distractors but no effect of "competition" $(F \mathrm{~S}<1.0)$ and no interaction $\left(F_{\mathrm{S}}<1.0\right)$.

A semantically related distractor hindered naming of targets from both large and small semantic neighborhoods. To further assess the effect of the semantically related distractor, $t$-tests for dependent samples were carried out for target words from both large and small neighborhoods. There was a significant difference between naming latencies with related and unrelated distractors in the condition with large neighborhoods [subject analysis, $t(23)=3.96, p<0.01$; item analysis, $t(26)=2.42, p<0.025]$ and in the condition with small neighborhoods [subject analysis,

${ }^{3}$ Semantically related distractors could not be matched for semantic distance to their targets ( 0.41 versus 0.23 ) because few distractors were available for low competition targets in the first place. However, the effects remained unchanged if semantic distance between target and distractor was included in the by-item analysis as a covariate ("distractor type": $F_{2}(2,102)=13.85, p<0.01$; "competition": $F<0.5$; distractor* competition: $F<0.7$ ). If the analysis was carried out using mixed effects modeling (Baayen et al., 2008), there were effects of Semantic Distance $(t=3.67)$, age of acquisition ( $t=3.28$ ), and length (letters: $t=3.20$, syllables: $t=-203$ ) but no effect of "competition" ( $t=-0.33)$, and no significant interaction $(t=-0.42)$. $t(23)=2.57, p<0.02$; item analysis, $t(26)=2.28, p=0.031]$. In contrast, if assessed with $t$-tests, no difference emerged between high and low competition items in the related distractor condition [subject analysis: $t(23)=1.47, p>0.15$; item analysis: $t(52)<1.0$ ].

It might be argued that some of the target-distractor pairs in the "low competition" condition were associatively related rather than being category co-ordinates ${ }^{4}$. Therefore, six target-distractor pairs were excluded (marked in the Appendix), and the data were subsequently re-analyzed. The remaining targets, again, did not differ in the relevant psycholinguistic variables (all $p$ 's $>0.12$ ). The results remained unchanged with a main effect of "distractor type" $\left[F_{1}(1.6,36.2)=76.96, p<0.01 ; F_{2}(1,40)=66.67, p<0.01\right]$ but no effects of "neighborhood size" $\left(F_{1}<1.0 ; F_{2}<1.0\right)$ and no significant interaction $\left[F_{1}(2,46)=1.5, p>0.20 ; F_{2}<1.0\right]$.

\section{Discussion}

Like Experiment 2, the present experiment revealed no main effect of a target item's neighborhood size upon naming latencies. The only significant main effect was of distractor type with a line of "xxxx" slowing down naming less than an unrelated distractor, and semantically related distractors causing more interference than unrelated distractors. The latter effect is well-established in the literature (Schriefers et al., 1990). The experiment was, thus, sensitive enough to detect differences in the interference condition. In contrast, naming latencies for target items from large and small categories were comparable. Across all three conditions, there was a difference of about $2 \mathrm{~ms}$ suggesting that there was not even a trend of a difference in the data. The failure to find a significant main effect was observed with a different experimental paradigm than Experiment 2 supporting the validity of the data. The interaction of both factors reached significance in the by-subject analysis but was not significant in the by-item analysis. It was, however, not in the direction predicted by the SLNH: this hypothesis suggests that related distractors should facilitate naming when few competitors (small cohorts) are activated (Abdel Rahman and Melinger, 2009a). For both groups of target items, however, a related distractor produced interference. The interaction was significant when the control condition ( $\mathrm{xxxx}$ ) was included in the analysis for which no model makes a prediction. When only related and unrelated distractors were considered in a $2 \times 2$ ANOVA, the interaction was not significant. Given that the main effect failed to reach significance in both experiments, the results do not support Abdel Rahman and Melinger's (2009a) theory.

\section{DISCUSSION}

Currently there is a debate whether lexical selection includes competition between a target lexical entry and semantically related competitors. This discussion was triggered by a number of results incompatible with the "selection-by-competition" account (Mahon et al., 2007; Mahon and Caramazza, 2009). Abdel Rahman and Melinger (2009a,b) have suggested modifications to the original account, the so-called SLNH. This new hypothesis suggested that the activation state of the whole cohort of activated competitors should be considered when evaluating lexical-SIE. Specifically, the account predicts a main effect of semantic neighborhood size: the more competitors activated, the more competition should result. In

${ }^{4} \mathrm{I}$ would like to thank Rasha Abdel Rahman for bringing this to my attention. 
addition, presentation of a distractor should produce interference when many competitors are activated while for few competitors, a printed distractor should facilitate word production.

These predictions were tested in three experiments. It was assessed whether naming pictures from large semantic categories (e.g., "tiger," "hammer") and small semantic categories (e.g., "funnel," "cage") would affect naming latencies. The target items were taken from a study of Bormann et al. (2008) and have been demonstrated to provoke different responses in anomic subjects. This finding was replicated in Experiment 1 which used a subset of target items and a different group of naming-impaired subject. Target items from large semantic categories caused semantic errors while target items from a small semantic category provoked errors of omissions. This provides strong evidence that, depending on semantic-lexical neighborhood size, many or few competitors were activated. Crucially in this study, both groups of items again yielded comparable rates of correct responses. Therefore, items in both groups were equally accessible for the impaired speakers.

The main effect of neighborhood size, which the SLNH predicts, was not observed in Experiment 2. Subjects named items in three consecutive cycles. A repetition effect, which is frequently observed (Barry et al., 2001; Belke et al., 2005), was replicated. Naming of pictures was slower during the first cycle than during the second and the third. However, there was no difference between target items from large and small semantic categories. The main effect as well as the interaction failed to reach significance.

In Experiment 3, semantic-lexical neighborhood size was combined with semantically related and unrelated distractors in a PWI paradigm. According to the SLNH, an interference effect should be observed for large semantic categories while a facilitation effect should be observed for small semantic categories (Abdel Rahman and Melinger, 2009a, p. 716). However, again no difference in naming latencies was found for items from large and small semantic neighborhoods. Naming latencies for these two groups of items were virtually identical. In addition, semantic interference was observed for both groups of items. Therefore, a semantically related distractor produces interference irrespective of the size of the activated network. The only significant factor in Experiment 3 was the type of distractor (semantically related versus unrelated). This latter effect is well-established in the literature (e.g., Schriefers et al., 1990). Note that the experiment is not reporting a null result: a significant interference effect was observed in a condition in which the SLNH predicts facilitation.

In sum, category size predicted the type of errors to be observed in a group of naming-impaired subjects. In contrast, the predicted effects of category size on naming latencies in two experiments were not observed while highly significant effects of repetition and of distractor type were. Caramazza and Costa (2001) had previously demonstrated that response set size did not affect naming latencies in PWI experiments. These observations are highly problematic for Abdel Rahman and Melinger's (2009a) hypothesis. One must conclude that the proposal, in its current form, receives no empirical support from the present study. Given that an effect of semantic category size is so central a prediction in their proposal, the authors may need to revise their central claim. However, this may also be of relevance for other models incorporating a comparable competition mechanisms, for example, the WEAVER model (Roelofs,
1992, 2001; Levelt et al., 1999; cf. Caramazza and Costa, 2001). On the other hand, Abdel Rahman and Melinger's (2009a) focus was on context-effects as, for example, provided in PWI and blocked naming experiments. One may argue that a context is required to create strong enough interference in the lexical network. Yet, conceptual facilitation and lexical interference are rather general processes which result from activation spreading independently of an explicit semantic context. One may, therefore, expect these to create measurable interference in other object naming tasks including those without explicit contexts. In addition, limiting the scope of the SLNH to "strong context" conditions would require several ad hoc assumptions. For example, one may assume that a distractor word introduces more overall activation to the lexical network. But this alone is not sufficient to determine the amount of interference as this further depends on the size of the activated cohort. How extra activation from a distractor would affect smaller and larger sets of competitors has not yet been specified.

A number of questions remain. For example, it has been left open how many competitors need to be active to generate measurable interference. Abdel Rahman and Melinger (2009a) discuss several different situations in which distractors activate a smaller set or a larger set. For example, "zebra" as distractor for "horse" (LSA semantic distance 0.13 ) is assumed to activate a smaller set of competitors while "whale" as distractor activates a much larger set (LSA semantic distance 0.0). It is, therefore, plausible to assume that net interference and facilitation effects will be a matter of degree rather than of a minimum number of co-activated lexical competitors. Yet again, below 3\% $(n=5)$ of responses were semantic errors in the low competition group. Thus, semantic errors were virtually absent for these targets. In contrast, $27.5 \%$ of responses were semantic errors in the high competition item group. This supports the view that fewer lexical competitors were available for low competition items. On the other hand however, facilitation through activation spreading in the conceptual network should be comparable in both conditions: according to the SLNH, spreading activation in the conceptual network involves associatively related concepts which is the standard view in semantic priming. Since targets from both conditions are part of a semantic network, they should both receive a comparable degree of facilitation through semantic priming. In fact, if near neighbors were determined for every target word using LSA (Landauer et al., 1998), no difference emerged in the semantic relatedness of the target and their neighbors (mean semantic similarity was 4.77 for high competition targets and 4.78 for low competition targets, $t<1.0, p>0.8)$. Thus, comparable facilitation through semantic priming should occur for both groups of target items. Yet, less competition but a comparable degree of semantic priming should lead to even larger net facilitation for the items with few competitors. Finally for the low competition items ("bridge"), few competitors exist in the first place. Thus, the activated network inevitably is very small. Exactly for this case, Abdel Rahman and Melinger (2009a) predicted semantic facilitation.

In their discussion of Abdel Rahman and Melinger's account, Mahon and Caramazza (2009) argued that the "Swinging Lexical Network" account further failed to explain a number of recent findings from their lab. For example, the hypothesis failed to account for the paradox finding of distractor word frequency (Miozzo and Caramazza, 2003). However, the approach of Abdel Rahman and 
Melinger (2009a) is but one modification of the original "selection-by-competition" assumption, and we note that the alternative proposal of Mahon et al. (2007) depends on additional and ad hoc assumptions to explain several recent findings. For example, no theory currently accounts for the paradox finding of semantic distance effects in interference paradigms. It has been demonstrated that related (same category) distractors cause more interference than unrelated (different category) ones. Yet, paradoxically, semantically close distractors interfere less than semantically dissimilar ("far") ones (Mahon et al., 2007). Rather than invoking activation dynamics, Mahon et al. (2007) assume a decision mechanism which has control over a limited-capacity response channel. For some reasons not yet specified this response mechanism is able to quickly exclude unrelated distractors from the response channel as well as semantically similar distractors but slow to remove semantically far distractors. Which criterion, if any, does the control mechanism adopt to generate this pattern of interference? This has not been worked out.

The results of Experiments 2 and 3 may also be discussed together with the results of Experiment 1. It was demonstrated that larger semantic neighborhoods provoke semantic paraphasias in subjects with anomia (cf. Blanken et al., 2002; Bormann et al., 2008). In contrast, unimpaired subjects were not affected by the number of lexical-semantic neighbors (see also Caramazza and Costa, 2001). This may suggest that unimpaired speakers may constrain the number of candidates in the lexicon early leading to a smaller number of competitors. Alternatively as in the model of Dell (1986), the selection process itself need not be a competitive process (cf. Mahon et al., 2007; Mahon and Caramazza, 2009). A model with thresholds (cf. Morton, 1984) could account for the available data although further work will be necessary to explore the strengths and weaknesses of this account. Upon presentation

\section{REFERENCES}

Abdel Rahman, R., and Melinger, A. (2009a). Semantic context effects in language production: a swinging lexical network proposal and a review. Lang. Cogn. Process. 24, 713-734.

Abdel Rahman, R., and Melinger, A. (2009b). Dismissing lexical competition does not make speaking any easier: a rejoinder to Mahon and Caramazza. Lang. Cogn. Process. 24, 749-760.

Baayen, R. H., Davidson, D. J., and Bates, D. M. (2008). Mixed-effects modeling with crossed random effects for subjects and items. J. Mem. Lang. 59, 390-412.

Baayen, R. H., Piepenbrock, R., and Van Rijn, H. (1993). The CELEX Lexical Database. Philadelphia: Linguistic Data Consortium.

Barry, C., Hirsh, K. W., Johnston, R. A., and Williams, C. L. (2001). Age of acquisition, word frequency, and the locus of repetition priming of picture naming. J. Mem. Lang. 44, 350-375.

Belke, E., Meyer, A., and Damian, M. F. (2005). Refractory effect in picture

of a picture for naming, semantically related lexical entries receive activation in parallel. In unimpaired speakers, the target entry will eventually receive activation above its threshold which triggers phonological encoding. The time to reach the threshold would be independent of activation states of other entries in the lexicon. In the case of paraphasias or slips of the tongue, noise in the system leads to another lexical entry candidate receiving above threshold activation upon which a semantically related competitor is selected for encoding. A model based on these assumptions could, thus, account for the difference between healthy and impaired speakers.

The difference between healthy and anomic speakers and between paradigms may also account for contradictory results in the literature: Mirman (2011) found that picture naming performance of aphasic speakers was facilitated by distant semantic neighbors. Vigliocco et al. (2002) reported stronger interference in semantically narrow contexts while Mahon et al. (2007) reported larger PWI through distant distractors. Clearly, further work is needed to understand the dynamics of lexical access as well as the locus of distractor induced interference. The recent discussion attests to the relevance of this issue to models of lexical access. The present study contributes to the issue by comparing directly picture naming of anomic and unimpaired speakers. Its primary goal, however, was to evaluate the SLNH which receives no support from the present experiments.

\section{ACKNOWLEDGMENTS}

The help of the participants is gratefully acknowledged. I would like to thank Rasha Abdel Rahman, Britta Biedermann, Gerhard Blanken, Brad Mahon, and Margret Seyboth for helpful comments on previous versions of this manuscript. I would like to thank Lars Konieczny for help with the linear mixed effects analysis in R. Two anonymous reviewers helped to improve the manuscript.

Caramazza, A. (1997). How many levels of processing are there in lexical access? Cogn. Neuropsychol. 14, 177-208.

Caramazza, A., and Costa, A. (2001). Set size and repetition in the picture-word interference paradigm: implications for models of naming. Cognition 80, 215-222.

Dell, G. S. (1986). A spreading-activation theory of retrieval in sentence production. Psychol. Rev. 3, 283-321.

Dell, G. S., and Gordon, J. K. (2003). "Neighbors in the lexicon: friends or foes?" in Phonetics and Phonology in Language Comprehension and Production: Differences and Similarities, eds N. O. Schiller and A. S. Meyer (New York: Mouton), 9-37.

Dell, G. S., Lawler, E. N., Harris, H. D., and Gordon, J. K. (2004). Models of errors of omission in aphasic naming. Cogn. Neuropsychol. 21, 125-145.

Ellis, A. W., Flude, B. M., Young, A., and Burton, A. M. (1996). Two loci of repetition priming in the recognition of familiar faces. J. Exp. Psychol. Learn. Mem. Cogn. 22, 295-308.
Ferreira, V. S., and Pashler, H. (2002). Central bottleneck influences on the processing stages of word production. J. Exp. Psychol. Learn. Mem. Cogn. 28, 1187-1199.

Finkbeiner, M., and Caramazza,A. (2006). Now you see it, now you don't: on turning semantic interference into semantic facilitation in a Stroop-like task. Cortex 42, 790-796.

Forster, K. I., and Forster, J. C. (2003). DMDX: a windows display program with millisecond accuracy. Behav. Res. Methods Instrum. Comput. 35, 116-124.

Goodglass, H., Kaplan, E., Weintraub, S., and Ackerman, N. (1976). The "tip-ofthe-tongue" phenomenon in aphasia. Cortex 12, 145-153.

Hodges, J. R., Patterson, K., Graham, N., and Dawson, K. (1996). Naming and knowing in dementia of Alzheimer's type. Brain Lang. 54, 302-325.

Howard, D., Nickels, L., Coltheart, M., and Cole-Virtue, J. (2006). Cumulative semantic inhibition: experimental and computational studies. Cognition 100 , 464-482. 
Janssen, N.,Schirm, W., Mahon, B.Z., and Caramazza, A. (2008). The semantic interference effect in the picture-word interference paradigm: evidence for the response selection hypothesis. J. Exp. Psychol. Learn. Mem. Cogn. 34, 249-256.

Kessler, B., Treiman, R., and Mullennix, J. (2002). Phonetic biases in voice key response time measurements. J. Mem. Lang. 47, 145-171.

Kittredge, A. K., Dell, G. S., and Schwartz, M. F. (2007). "Omissions in aphasic picture naming: AoA is the culprit, as well as low semantic density," in Poster Presented at the 45th Meeting of the Academy of Aphasia, Washington, DC.

La Heij, W., and van den Hof, E. (1995). Picture-word interference increases with target-set size. Psychol. Res. 58, 119-133.

Laine, M., and Martin, N. (1996). Lexical retrieval deficit in picture naming: implications for word production models. Brain Lang. 53, 283-314.

Lambon Ralph, M. A., Graham, K. S., Ellis, A. W., and Hodges, J. R. (1998). Naming in semantic dementia what matters? Neuropsychologia 36, 775-784.

Landauer, T., Foltz, P. W., and Laham, D. (1998). Introduction to latent semantic analysis. Discourse Process. $25,259-284$

Levelt, W. J. M., Roelofs, A., and Meyer, A. S. (1999). A theory of lexical access. Behav. Brain Sci. 22, 1-75.

Mahon, B. Z., and Caramazza, A. (2009). Why does lexical selection have to be so hard? Comment on Abdel Rahman and Melinger's swinging lexical network proposal. Lang. Cogn. Process. 24, 735-748.

Mahon, B. Z., Costa, A., Peterson, R. Vargas, K., and Caramazza, A. (2007) Lexical selection is not by competition: a reinterpretation of semantic interference and facilitation effects in the picture-word interference paradigm. J. Exp. Psychol. Learn. Mem. Cogn. 33, 503-535.

Miozzo, M., and Caramazza, A. (2003). When more is less: a counterintuitive effect of distractor frequency in picture-word interference paradigm. $J$. Exp. Psychol. Gen. 132, 228-252.

Mirman, D. (2011). Effects of near and distant semantic neighbors on word production. Cogn. Affect. Behav. Neurosci. 11, 32-43.

Morton, J. (1984). "Naming," in Dysphasia eds S. K. Newman and R. Epstein (London: Churchill Livingstone), 217-230.
Navarrete, E., Mahon, B. Z., and Caramazza,A. (2010). The cumutative semantic cost does not reflect lexica selection by competition. Acta Psychol. (Amst.) 134, 279-289.

Roelofs, A. (1992). A spreading-activation theory of lemma retrieval in speaking. Cognition 42, 107-142.

Roelofs, A. (2001). Set size and repetition matter: comment on Caramazza and Costa. Cognition 80, 283-290.

Schnur, T. T., Schwartz, M. F., Brecher, A. and Hodgson, C. (2006). Semantic interference during blocked-cyclic naming: evidence from aphasia. $J$. Mem. Lang. 54, 199-227.

Schriefers, H., Meyer, A. S., and Levelt, W. J. M. (1990). Exploring the time course of lexical access in language production: picture-word interference studies. J. Mem. Lang. 29 86-102.

Stemberger, J. P. (2004). Neighborhood effects on error rates in speech production. Brain Lang. 90, 413-422.

Stroop, J. R. (1935). Studies of interference in serial verbal reactions. J. Exp. Psychol. 18, 643-662.

Vigliocco, G., Vinson, D. P., Damian, M F., and Levelt, W. (2002). Semantic distance effects on object and action naming. Cognition 85, B61-B69.
Vitevitch, M. S. (2002). The influence of phonological similarity neighborhoods on speech production. $J$. Exp. Psychol. Learn. Mem. Cogn. 28, 735-747.

Conflict of Interest Statement: The author declares that the research was conducted in the absence of any commercial or financial relationships that could be construed as a potential conflict of interest.

Received: 17 December 2010; accepted: 31 May 2011; published online: 09 June 2011.

Citation: Bormann T (2011) The role of lexical-semantic neighborhood in object naming: implications for models of lexical access. Front. Psychology 2:127. doi: 10.3389/fpsyg.2011.00127

This article was submitted to Frontiers in Language Sciences, a specialty of Frontiers in Psychology.

Copyright (c) 2011 Bormann. This is an open-access article subject to a nonexclusive license between the authors and Frontiers Media SA, which permits use, distribution and reproduction in other forums, provided the original authors and source are credited and other Frontiers conditions are complied with. 
APPENDIX

Target words used in the three experiments.

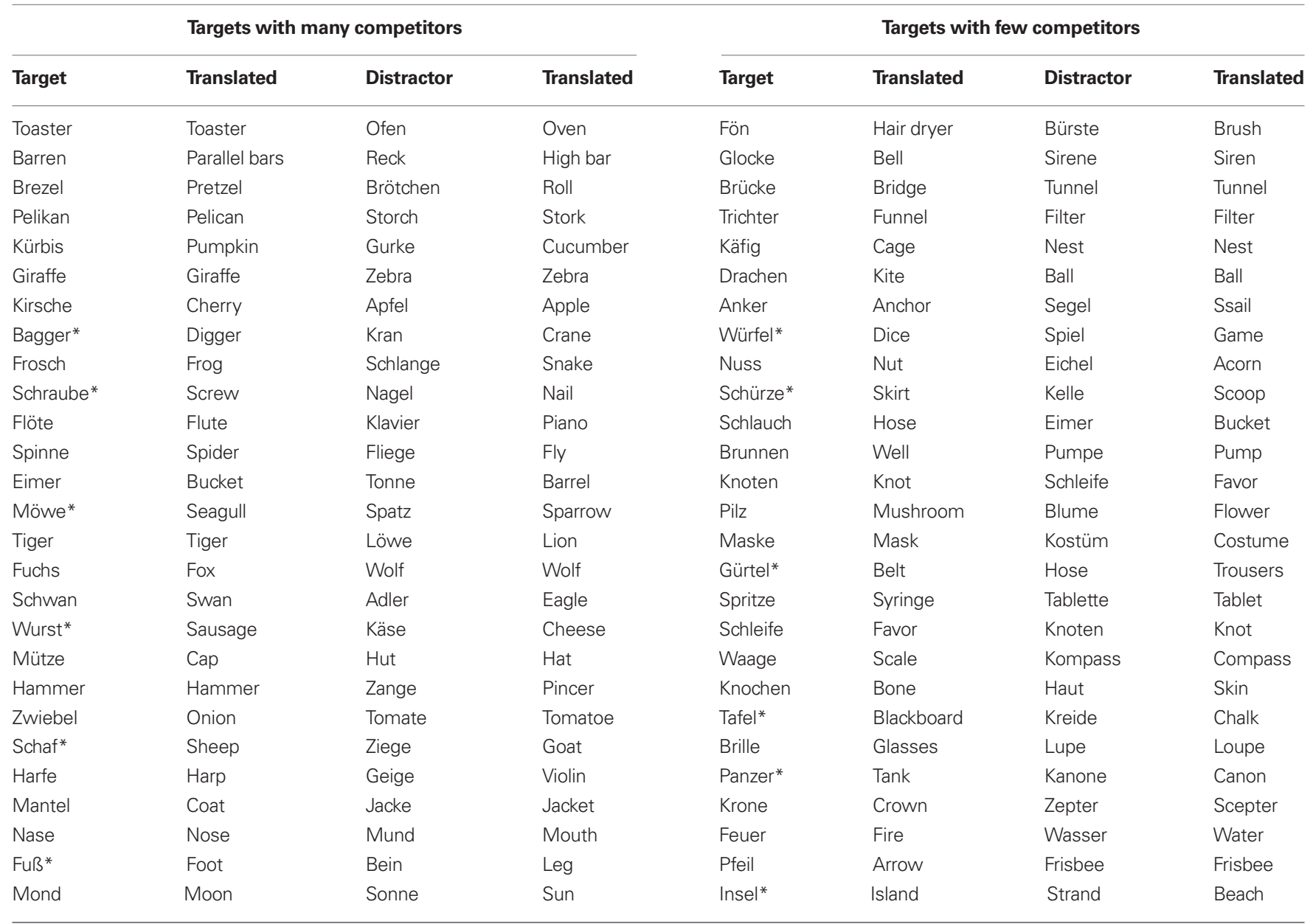

*Excluded for re-analysis of Experiment 3 (see text for details). 